\title{
Balancing Tensions between Caregiving and Parenting Responsibilities in Pediatric Patient Care
}

\author{
WOOSUK SEO, University of Michigan, USA \\ ANDREW B.L. BERRY, Kaiser Permanente Washington, USA \\ PRACHI BHAGANE, University of Michigan, USA \\ SUNG WON CHOI, University of Michigan, USA \\ AYSE G. BUYUKTUR, University of Michigan, USA \\ SUN YOUNG PARK, University of Michigan, USA
}

In pediatric chronic care, the treatment process affects not just the child's physical health, but his or her psychosocial and emotional development. As a result, caring for pediatric patients with a chronic illness such as cancer is becoming a daunting task for parental caregivers. They are expected to fulfill the caregiving needs of managing the child's health condition and treatment while also meeting the parenting needs of translating knowledge, communicating about the illness, and making numerous decisions on a daily basis for their sick child due to the child's young age. Drawing on 15 semi-structured interviews, we examined parental caregivers' perspectives on raising a child while also managing the child's health. We identified three tensions that participants encountered as they balanced parenting and caregiving responsibilities: (i) tension between ensuring the child's health and safety and attending to the child's social development, (ii) tension between disclosing health-related information and minimizing the psychological burden on the child, and (iii) tension between rewarding the child's cooperation in treatment and maintaining discipline. Together, these tensions reveal an ongoing process through which caregivers assess and interpret their actions and responsibilities relative to anticipated consequences across multiple time scales. These findings reveal opportunities for sociotechnical systems to account for and support this active process of iterative cycles of assessment.

CCS Concepts: • Human-centered computing $\rightarrow$ Empirical studies in HCI; • Applied computing $\rightarrow$ Health care information systems.

Additional Key Words and Phrases: caregiver; parent; responsibility; pediatric patients; chronic illness

ACM Reference Format:

Woosuk Seo, Andrew B.L. Berry, Prachi Bhagane, Sung Won Choi, Ayse G. Buyuktur, and Sun Young Park. 2019. Balancing Tensions between Caregiving and Parenting Responsibilities in Pediatric Patient Care. Proc. ACM Hum.-Comput. Interact. 3, CSCW, Article 153 (November 2019), 24 pages. https://doi.org/10.1145/3359255

\section{INTRODUCTION}

When a child is diagnosed with a debilitating chronic illness, such as cancer, parents face the challenge of having to re-define their identity as caregivers and adjust to new responsibilities [15].

Authors' addresses: Woosuk Seo, University of Michigan, Ann Arbor, USA, seow@umich.edu; Andrew B.L. Berry, Kaiser Permanente Washington, Seattle, USA, ablberry@uw.edu; Prachi Bhagane, University of Michigan, Ann Arbor, USA, bhagane@umich.edu; Sung Won Choi, University of Michigan, Ann Arbor, USA, sungchoi@med.umich.edu; Ayse G. Buyuktur, University of Michigan, Ann Arbor, USA, abuyuktu@umich.edu; Sun Young Park, University of Michigan, Ann Arbor, USA, sunypark@umich.edu.

Permission to make digital or hard copies of all or part of this work for personal or classroom use is granted without fee provided that copies are not made or distributed for profit or commercial advantage and that copies bear this notice and the full citation on the first page. Copyrights for components of this work owned by others than ACM must be honored Abstracting with credit is permitted. To copy otherwise, or republish, to post on servers or to redistribute to lists, requires prior specific permission and/or a fee. Request permissions from permissions@acm.org.

(c) 2019 Association for Computing Machinery.

2573-0142/2019/11-ART153 \$15.00

https://doi.org/10.1145/3359255

Proc. ACM Hum.-Comput. Interact., Vol. 3, No. CSCW, Article 153. Publication date: November 2019. 
These often include providing additional physical care to the sick child, monitoring treatments, overseeing medication adherence, communicating with clinicians, and coordinating care-related activities. However, because of the child's young age, parents must manage these new caregiving responsibilities alongside traditional parental responsibilities, such as guiding the child's social development, participating in her/his education, and supporting her/his emotional well-being. Thus, for parental caregivers, caring for pediatric patients becomes a daunting task of fulfilling competing responsibilities and expectations.

Previous CSCW and HCI studies have examined the needs of parental caregivers in a variety of healthcare contexts such as treating diabetes, asthma, autism or cancer, and have explored ways of meeting these needs through technological support. They have shown the potential for social computing technologies to help patients and caregivers manage chronic conditions [18, 31, 40] and to provide physical, social, and emotional support [15, 21, 22]. These systems have largely focused on caregiving for health needs, such as helping coordinate care endeavors [5] and providing convenient means for caregivers to help patients. Our study, however, focuses on the caregivers' struggles and efforts to address other essential responsibilities that come with taking care of their chronically ill child. In particular, we focus on how caregivers manage and carry out dual responsibilities as parents and caregivers in daily life.

In this study, we aim to understand how to better support the needs of parents who are caregivers for young children (ages 6-12) with cancer. We have found that caregiving work [36] in which parents support a child through the chronic care process often creates tension with their everyday parental roles - i.e., am I being a good caregiver and also a good parent to my child? These tensions raise unique challenges for parents and add significantly to their burden of care since the needs created by two different sets of responsibilities are not always aligned in everyday life. We show that CSCW technologies must be designed with an understanding of such tensions in order to more effectively support parental caregivers.

It has been previously noted that "the parental role is inherently a caregiver role" [12]. We do not dispute this fact and our goal is not to draw an artificial distinction between parenting and caregiving. In this study, we make an analytic distinction between the two to help us convey the complexities and nuances of the work that should be supported by system design. Kaziunas et al. [15] made a similar distinction in showing that parental caregivers to pediatric cancer patients must adapt to the new social world of the hospital and to the "life of having a sick child," and that this transition from parent to caregiver places an additional emotional burden on the information work which is necessary to manage the illness. They have shown that parents have to navigate multiple social worlds as they move between home and clinic. Our study extends this work by focusing on the tensions that parents encounter due to conflicting responsibilities of parenting and caregiving. It reveals opportunities for systems to help caregivers balance these tensions through reflection, action, and reassessment over time, and to help them manage treatment trajectories and parenting trajectories together.

In this paper, we report findings from a qualitative field study involving semi-structured interviews with 15 parents who are caregivers for their young children with cancer. We identify what specific responsibilities they carry as parental caregivers, the kinds of tensions they perceive and face in carrying out parental and caregiving responsibilities, and the process of decision-making parental caregivers perform to balance these tensions. Based on our study and analysis, we make the following contributions:

- We illustrate three tensions that participants perceived between parental and caregiving responsibilities, thereby extending the literature on the needs of this specific population. 
- We discuss how parental caregivers carry out a constant process of assessing, acting, and re-assessing to balance co-occurring parental and caregiving responsibilities across different time scales.

- We present specific design implications for sociotechnical systems to support parental caregivers in balancing parental and caregiving responsibilities in pediatric care.

In the next section, we first present relevant work from the existing literature. We then describe our methodology, which includes a brief description of the context of our study, before presenting the findings.

\section{RELATED WORK}

\subsection{The role and dilemma of parental caregivers}

Previous studies in the medical literature have looked at the experience of parental caregivers in pediatric patient care (e.g., [7, 12, 43]). These studies examined the role and responsibilities of caregivers of children with chronic illness and its impact on parental caregivers. For instance, Young and the colleagues $[43,44]$ showed that after receiving a diagnosis, mothers of children with cancer had to carry out new responsibilities, including caregiving tasks (e.g., being physically close to children, managing children's cooperation with treatment) and technical tasks (e.g., acting as "brokers" of information for their children). They pointed out that these obligations result in role strain by compromising parents' ability to carry out other roles, including being a parent of their other children. Their analysis revealed parents' struggles in managing the task of raising their children to be more independent and the task of supporting the children with special needs. Similarly, Jones [12] described a dilemma that parental caregivers experience between their obligation to provide painful care and treatment and their desire to comfort and help their child avoid suffering such pain.

We also identified different parenting styles of caregivers in the literature. Drawing on Regulatory Focus Theory (RFT), Eiser et al. [7] analyzed two different parenting styles of parents of cancer patients: promotion-focused (the focus on approaching positive outcomes) and prevention-focused (the focus on avoiding negative outcomes). Based on their analysis, the authors found that parents reported more promotion than prevention and that quality of life for children with cancer is related to prevention focus. However, regardless of the parenting styles, the parents experienced a dilemma in which they wished to encourage the patients to fulfill achievements that would be expected under normal circumstances, but soon realized that such activities might cause risks of illness [7]. From a broader perspective, even parents of healthy children find potential conflicts with other situated factors in their daily lives, such as conflicts between various parenting styles and technology use [27]. Nonetheless, having an additional responsibility of caring for chronic health conditions of their children adds more complexity into the situated parenting practices.

While these studies examined the dilemmas parental caregivers experienced, most of them focus more on describing caregiving tasks than on generalizing aspects of parenting in pediatric chronic health management. Little is known about which factors of caregiving and parenting cause such dilemmas, how caregivers come to face them, how they think through them, and what they do to resolve them. These studies put their weight toward implications for clinical practices and offer few implications for designing technical systems to help caregivers manage their dilemmas.

\subsection{Supporting parental caregivers' work in CSCW}

Researchers in the CSCW and HCI communities have examined parental caregivers' work in terms of their roles, impacts on their lives, their psychological burdens and their interactions with health providers $[18,22,24,28,39,41]$. Although previous studies explored parental caregivers' 
experiences in a variety of healthcare contexts, such as caring for children with diabetes (e.g., [13]) or autism (e.g., [26]), these studies mostly focused on supporting pediatric patients (e.g., improving medication adherence) or supporting caregivers' tasks (e.g., providing convenient means for caregivers to help patients, or facilitating more effective communication with providers for their child patients). Prior works have not considered how parental caregivers navigate potential conflicts between the responsibilities of being a parent and those of being a caregiver. Unlike caring for mature family members as an informal caregiver (e.g., caring for a spouse) in which patients and caregivers each negotiate tensions involving healthcare needs, personal values, and the demands of daily life [3], parents may take on a majority of necessary care responsibilities and only share the resulting stressors with children selectively [16]. Thus, there is still a critical need to understand how parental caregivers perceive and work through tensions that emerge in caring for pediatric patients with chronic illness.

A few studies focus on parental caregivers' experience as caregivers while managing their own lives (e.g., [13, 23, 25]). An important study is the work by Kaziunas et al. [15], which examined the interconnections between information and emotional work during the transition from parent to caregiver. Their findings showed how caregivers engaged with multiple logics around alternative therapies. Families often understood these competing logics, but clinicians did not. One of these logics was related to the parenting experience. In this case, caregivers worried about the impact of treatment on their children not just medically, but also on the children's quality of life. For instance, a father recognized his son's desire for the normal life that he used to have, such as having a dog or going back to school, while he could not meet these needs due to a clinical protocol. Although Kaziunas et al. emphasize the importance of considering parental caregivers' engagement with multiple logics, there is a paucity of data about how such engagement causes tensions between caregiving and parenting logics.

Designing and developing technologies to support parents as informal caregivers has also received significant attention in recent years. Prior work suggests interventions to address the challenges of parental caregivers in various healthcare contexts, such as wearable cameras to gain a better understanding of the needs of children with autism [26] or educational games for children with diabetes [20]. These interventions also support caregivers in tracking and monitoring the patient's health status and medication (e.g., [33, 42]), facilitating social support for caregivers (e.g., [8, 31]) and navigating information about the children's illness and treatment trajectory (e.g., $[2,11,21])$ However, current interventions for parental caregivers are heavily weighted to provide support in managing patients' health and carrying out caregiving tasks; managing responsibilities between caregiving and parenting is not understood or supported yet. Building on this line of work in CSCW, we aim to uncover opportunities for sociotechnical interventions to support parental caregivers in managing dilemmas between caregiving and parenting responsibilities. We focus on caregiving practices in parental caregivers' daily lives after discharge from the hospital. We seek to extend previous work (e.g. $[6,15])$ to understand the process through which caregivers understand and balance their two sets of responsibilities.

\section{METHOD}

\subsection{Data collection}

We conducted semi-structured interviews with 15 caregivers of children (ages 6-12) with cancer over one year. All interviewees identified themselves as the primary caregivers to the ill children, therefore carrying the greatest caregiving responsibilities in their family. The study was approved by our university's Institutional Review Board (IRB) for Medical Research prior to data collection. Interview participants were recruited at an outpatient oncology clinic for pediatric care. We 


\begin{tabular}{ccccc} 
Participant & Relationship & Patient Age & Going to School & Digital device for patient \\
\hline$*$ CG1 & Mother & 6 & Yes & Tablet \\
CG2 & Mother & 8 & No & Tablet \\
CG3 & Mother & 9 & Yes & Tablet \\
CG4 & Mother & 6 & Yes & Tablet \\
$*$ CG5 & Mother & 10 & Yes & Tablet \\
$*$ CG6 & Mother & 7 & Yes & Tablet \\
CG7 & Mother & 7 & Yes & Tablet \\
$*$ CG8 & Mother, Stepfather & 6 & Yes & Tablet \\
$*$ CG9 & Father & 9 & Yes & Tablet, Phone \\
CG10 & Mother & 12 & Yes & Phone \\
CG11 & Mother, Father & 12 & No & Phone \\
CG12 & Mother & 9 & Yes & Tablet \\
CG13 & Father & 6 & Yes & Tablet \\
CG14 & Grandmother** & 6 & Yes & Phone \\
CG15 & Father & 7 & Yes & Tablet
\end{tabular}

Table 1. Demographic information of the interview participants and their patients. * Caregivers of pediatric patients who were diagnosed at the young age of 8 months -3 years old $* *$ P14's parents are divorced and have their own households. Thus, CG14 takes on the parenting role too.

approached and asked parents if they were primary caregivers to pediatric patients and if they would be willing to share this experience for a study. Pediatric patients had to meet the following criteria for inclusion: 1) they were diagnosed with cancer at least two months prior and 2) they were between 6 and 12 years old. This allowed us to focus on pediatric patients who had already experienced the treatment process after initial diagnosis and needed a significant amount of caregiving from their parents due to their young age. Based on these criteria, we identified more than 40 eligible caregivers; however, only 15 of them agreed to take part in our study. Among about 25 caregivers who did not participate, a few declined due to unknown personal reasons. The majority were interested in participating but could not do so due to attention needed for the child's weak health condition, sudden schedule changes based on lab test results (e.g., being admitted), and immediate time constraints such as having to drive their child back to school after the clinic visit.

Each interview took 40-60 minutes, either in a consultation room or in the infusion unit if the patient was receiving treatment. Semi-structured interview questionnaires were used, focusing on understanding the caregivers' lived experience of carrying out and navigating their caregiving work for the child's treatment. Specific questions pertained to parent-child interactions before and after the diagnosis, communicating with the child about their illness, managing the illness in everyday life, and the child's responses to their illness and treatment. (e.g., sample questions included, "How do you communicate about the illness with your child?" "In your opinion, what are the most challenging aspects for your child in terms of interacting with friends?")

All interviews were audio recorded and later transcribed. We removed all personally identifying information and assigned a unique identifier to each participant. In some cases, both parents were willing to participate in the interview. In these cases, we treated both parents as one pair of caregivers and interviewed them together (e.g., CG11 indicates both mother and father unless they are labeled separately). The participants include 9 mothers, 3 fathers, 2 couples, and 1 grandmother (See Table 1). 


\subsection{Data Analysis}

The study team of four members individually coded the first three interview transcripts to identify central themes. Affinity diagrams [4] were used to discuss and identify initial themes that commonly occurred. We identified different categories of challenges that caregivers experienced in the course of treatment. This helped us to focus subsequent interviews. Three members of the team coded the rest of the interview transcripts in an iterative process that accounted for any new themes. During this process, the study team met regularly to discuss the findings and identified emerging themes regarding tensions between responsibilities. Axial coding was done to identify any relationships between themes [35]. The themes produced by each researcher were compared, discussed and revised through a series of discussions until agreements were reached. This study is still in progress. In this paper, we report our initial findings about tensions between parenting and caregiving responsibilities for caregivers of pediatric cancer patients.

\section{MEDICAL CONTEXT}

In this study, we focus on the experiences of caregivers of pediatric patients who have undergone either chemotherapy or bone marrow transplant (BMT) for blood cancer or disorder. BMT involves both a high dose of chemotherapy and the infusion of stem cells that produce new blood cells. Side effects and complications may occur from both chemotherapy and transplant, some of which may be life-threatening; because the immune system is suppressed, the patient is at high risk for infection. BMT patients may be kept on medications that suppress the immune system for many months, even years. This is in partly to prevent what is known as graft-versus-host disease (GVHD), in which donor cells recognize the patient's body as foreign and attack it. Since GVHD may occur in multiple organ systems and range from mild to fatal, patients are followed and monitored very closely by their clinical team. Patients usually stay in the hospital for several weeks following transplant, after which they are seen in the clinic at least once a week, and then once every other week, and then with decreasing frequency over time. Given the considerable risk of serious and potentially life-threatening complications, caregivers and patients need to manage the complex treatment trajectory jointly with the clinicians. In pediatric care, most of this work is done by parental caregivers. Especially in the first year after transplant, the caregiver has multiple responsibilities that include managing clinical visits, administering the child's numerous medications, monitoring various symptoms, and practicing infection prevention. In the next section, we present three cases where parents' caregiving responsibilities come into tension with their everyday parenting responsibilities.

\section{FINDINGS}

Our analysis generated three themes regarding tensions perceived by parents of pediatric cancer patients. These tensions manifested as parents grappled with new caregiving responsibilities amidst the ongoing responsibilities of parenting. In the following sections, we discuss tension between keeping the child physically healthy and attending to the child's social development, tension between sharing health information and minimizing psychological burdens on the child, and tension between facilitating cooperation with treatment and maintaining discipline.

\subsection{Tension between keeping the child physically healthy while attending to their social development}

All of the caregivers in our study perceived tension between two needs: keeping the patient physically healthy (e.g., by minimizing exposure to infection) and attending to the patient's social development. From a medical perspective, one of the caregivers' critical responsibilities was to 
minimize their child's exposure to potential sources of infection. Most patient participants had received bone marrow transplants (BMT) as part of treatment. As we described above, the patient typically takes medication to weaken the immune system deliberately to minimize the risk of GVHD. However, weakening the immune system means that any infection could be life-threatening Thus, caregivers have to ensure that the patient is not exposed to any potential sources of infection until their health condition stabilizes. This period of highest risk could last up to a year or more.

To avoid risks of infection, caregivers came up with various extreme health management protocols. They maintained a completely sterile environment at home by minimizing furniture and other physical artifacts in their house, constantly wiping surfaces, and washing bedding sheets and pillowcases every day. They also kept patients isolated from public spaces. For instance, all the patients in our study stayed at home for several months to a year since other children could be potential sources of infection, as even a simple handshake with a friend with a cold might result in infection and threaten the patient's life. Thus, the caregivers had to restrict patients' social interactions with their peers, and most did not send their child to school for up to a year to ensure the child's safety. However, making sure the patient was safe from potential infection risks resulted in social isolation for the child. Caregivers realized that while isolating a child at home fulfilled caregiving responsibilities, it did not meet the child's social needs and negatively affected the child's social development. Caregivers also perceived that it was challenging for patients to be completely separated from their pre-existing social groups.

Thus, keeping the child physically healthy (preventing infection) and fulfilling the child's social needs became a serious dilemma for caregivers. On one hand, from the perspective of a parent, they considered that such social interaction would be or was critical for the child's personality and social skill development. Caregivers observed how interactions with peers, siblings, and others affected the mood of their children and the development of their personality and social skills. In particular, caregivers noticed the need for social interactions when their child was kept isolated due to health concerns. Many told us that they had a hard time comforting their child when he or she wanted to go outside with friends or looked depressed. On the other hand, from the perspective of a caregiver, they worried that the patient's physical interactions with others, such as hugging or shaking hands, could result in infection. CG2 described the challenge for her and the patient in this regard:

"The hardest part for him [P2] was- during transplant, at the end, when he had not seen his brother, sister and for weeks he had been into this room for weeks and that's when we saw him wear down. And maybe he would say that this couple of months away from friends and being home with me every day. We get along very well. We are pals, but I am not his friend, not his social outlet. " - CG2 (Mom)

The caregiver in the quote observed the impact of isolation on P2 during his hospital stay. While the caregiver tried to be with P2 to meet his social needs, she soon realized that she could not replace interacting with his friends. Our interviews suggest that while the effect of isolation on social needs and social growth was not initially visible to caregivers, they started noticing it over time. Accordingly, they were concerned that limiting their children's social interactions would affect their growth and development negatively, particularly since they were still young and supposedly learning not only knowledge but also social skills and norms through interactions with and observations of peers in everyday life. For instance, CG6 described how P6 missed out on going to kindergarten frequently due to treatment, which hindered P6 from developing social skills and having age-appropriate social interactions:

"In kindergarten, she [P6] missed still quite a bit because we were coming every six weeks. I was also a little nervous because we're on our new system not bouncing back yet. When anything was going around the school, I would just keep her home. I know it's bad, but I felt like I could handle it. (...) This year was a great year for her. Even the teacher felt like most of the growth that she made was social 
growth that needed ... Would have occurred probably in preschool and kindergarten, but because she wasn't present very often there..." - CG6 (Mom)

This quote shows that the caregiver noticed the child's social development happened mostly after the child returned to kindergarten. She also received feedback about the child's social growth from the kindergarten teacher, which could crucially help gauge and assess her child's development progress. Not only does keeping the child home from school prevent significant social interactions for her age, but it also removes an important mechanism and opportunity for the caregiver to gauge, evaluate, and confirm their child's social development.

We also found that caregivers had difficulty balancing needs when their child had already learned social skills and norms but did not understand the infection risks associated with social interaction. For example, patients wanted to show affection and friendship through behaviors like hugging because they believed these friendly behaviors were part of normal social interaction. In this case, caregivers intentionally had to work against these learned norms to keep their children from coming into physical contact with others. For instance, CG3 described having a hard time because their child wanted to hug his friends at school, which was good behavior but risky for his health:

"And for a long time, since he [P3] is being born we always hug him and kiss him and love him. So, he is a very physical loving child. We had a really, very hard time when he went to school. We knew he shouldn't really hug kids all the time. And we had really big issues with him wanting to hug them. I would get upset because he is not hitting, he is not kicking, but he just wants to hug. He just wants to be nice...” - CG3 (Mom)

The quote illustrates how CG3 struggled with a dilemma where she had to work against what she believed to be good and important. She had been raising her child with particular social skills, but after the diagnosis she had to prevent him from doing what she had raised him to do, which caused the dilemma. This issue was not apparent until the child started going to school and making friends. In the quote, CG3 emphasized how difficult it was to keep the child healthy by not allowing what he was taught to do to be friendly.

The tension around social needs had ramifications beyond the patients' social interactions and development. It also affected the immediate family's relationships with extended families, family friends, and others. For instance, CG12 described challenges she faced at family gatherings:

"I've been in that position many times with my own family members bringing sick kids over and then I have to make the choice. Am I going to take him out of our Christmas celebration when he was looking forward to it for months and hasn't seen anyone because we were so isolated when he's vulnerable, like that. And it's this terrible position to put him in and me in and people just don't seem to get it, even family doesn't get it. I'm always amazed..." - CG12 (Mom)

In this case, CG12 had to make a choice whether to take P12 out of a Christmas celebration involving sick relatives after P12 had looked forward to it for months. CG12 was frustrated to be in the position of having to make this choice, and perceived that other family members did not understand her situation. In her mind, if other family members had understood P12's situation, they would have not brought their sick children to the family gathering. Like CG12, many caregivers in the study mentioned that the issue with the social isolation sometimes went beyond the child and affected the entire family, which eventually could lead to family isolation.

To deal with the tension between addressing social needs and keeping the patient from potential infection risk, some caregivers provided mobile communication tools, such as smart phones or iPads, for their patients as an alternative. Caregivers considered that these mobile tools aided in alleviating the problem with social isolation by connecting patients virtually with their peers, even though getting them these fancy devices was against their ideal parenting style. For example, CG11 explained why he bought P11 a phone, despite thinking a phone was not necessary for children of P11's age:

Proc. ACM Hum.-Comput. Interact., Vol. 3, No. CSCW, Article 153. Publication date: November 2019. 
"We got him a phone that he never had before. We weren't going to get him a phone as young as he was, but once he was diagnosed we got him a phone so he can keep contact with some of his closest friends that way. So, he does too call and text back and forth with some of the closest ones. But he's definitely looking forward to being able to go back to school hopefully" - CG11 (Dad)

Here, the phone indicated the evidence of CG11's attempt to meet P11's social needs. By emphasizing that he would not have bought P11 a phone if he was not isolated due to the treatment, CG11 acknowledged the importance of social interactions to P11. He also carefully shifted his parenting attitude and expectations he kept in response to caregiving responsibilities by breaking a rule of his own discipline regarding educating his child. Initially, CG11 recognized a phone as a fancy device for a child. Later, he saw it as a tool that would keep P11 within his social group. Although the limited, virtual interaction over the smart phone would not be the solution to social isolation, CG11 was satisfied to provide at least a partial social outlet for P11.

Another strategy for meeting social needs while keeping a patient safe was to communicate with their school teachers closely. Since caregivers could not really be with their patients, especially when the patients were at school, all of our caregivers tried to communicate about their patients' condition and requested necessary help from teachers. For instance, a teacher set a medication reminder on her phone to help a child get into the routine (P3), and observed the child's health condition to get special attention when needed (P13) or kept him/her away from any kid who seemed sick (P10). By sharing the health status of the patients, caregivers expected teachers to help carry out some of the caregiving responsibilities. CG10 reflected on how P10's teachers cooperated with her request:

"If he[P10] sees somebody sniffling or even coughing, he observes it and sees, is that cough because they have something stuck in their throat or is that a sniffle because it's just a sniffle or is he sick? He's pretty observant and if he notices that, the teachers are all aware of it so they know that if he looks at 'em and gives them that look, like, I need to move or get out of the classroom, they just allow him to do it." - CG10 (Mom)

This case of CG10 shows that teachers' cooperation in managing P10's health. Keeping CG10 away from the risk of infection was a caregiving task that CG10 would have done if she was there, but CG10 shared this task with the teachers. However, several caregivers also pointed out the difficulty in getting consistent support from their teachers. Some teachers might react differently to the patient's request by not being attentive, not being physically around the patient, or not communicating with other teachers. Thus, to maximize help, parents were still required to make an active effort to keep informing and reminding teachers of specific needs and requests.

In other cases, a few caregivers tried to bring in their clinicians to find a more reasonable solution that might help the patient understand. CG2 shared her experience with coming up with a reasonable timeline to go back to school when P2 complained about homeschooling:

"Last time, we were at home, working on school stuff. And he was like, I just want to be in school, I don't want to do it here. And what I said was, Okay! This is the situation we are in. Let's talk to [the doctor] and get a timeline when you can go back. What he says is a final." - CG2 (Mom)

Here, when CG2 realized the difficulty in finding out what would be a good, reasonable timeline that the child could aim to go back to school again, since things were still up in the air for him, she tried to bring the clinician into the conversation, as someone who had more expertise and could give the child some anchor point to aim for. Similar to how caregivers used or relied on feedback from teachers, caregivers emphasized doctors' role as the person who had the right expertise to provide solutions or make the final decisions, such as the expected, reasonable timeline to go back to school.

To summarize, we found that parental caregivers perceived tension between assuring their child's health safety and ensuring their child's social development. When the caregiver understood 
infection prevention to be a higher priority than social interaction, the child's social needs were often not fulfilled. To mitigate the tension, caregivers tried to use coping strategies such as requesting help from teachers, using expert opinions of clinicians, and mobile communication technologies, though these sometimes might be counter to the caregiver's parenting style.

\subsection{Tension between sharing health information and minimizing psychological burden on the child}

Caregivers perceived a tension between sharing health-related information with their child and minimizing their child's psychological distress. Due to the patients' young age, caregivers typically assumed complete responsibility for understanding the patient's diagnosis, prognosis, and other health-related information. Caregivers learned this information through interactions with healthcare providers and from other sources, such as researching on the Internet. As a result, caregivers were in a position to share health-related information with patients or withhold it. Caregivers often faced a choice regarding which information or how much information to share. For example, some caregivers explained the bone marrow transplant procedure and which medications to take each day. We encountered a range of approaches to sharing information about the child's illness. First, we will describe how some caregivers shared everything with patients to respect the patient's right to know and encourage the patient's self-management. Then we will share examples in which caregivers chose selectively to share or not to share information when they perceived that this information would be psychologically burdensome for the patient. These examples will illustrate the tension between sharing information and minimizing psychological burdens.

\subsubsection{Encouraging Self-Management through Full Information Disclosure. Some caregivers shared} everything about illness with patients. These caregivers believed that the patients had the right to know about their illness and treatment. Additionally, these caregivers wanted to raise their children to be ready to self-manage their illnesses independently. Thus caregivers communicated all health-related information, even the life-threatening nature of cancer. For instance, CG6 mentioned that open, transparent communication could empower her daughter to better prepare and go through the treatment process with confidence and awareness:

"So, I try to be as open in good communication with her as possible. I feel like it gives her empowerment if she knows everything. I always say the forest before the trees. Just give her the forest then it's easier for her to see the trees. Giving her as much information as possible, at her level, you know, developmentally appropriate level, and then having her move forward with as much confidence or awareness as she can" - CG6 (Mom)

In this quote, CG6 described her information disclosure about the illness and treatment as the forest and the trees. The forest would be the trajectory of treatment, while the trees would be small, daily health management activities, such as washing hands. According to CG6, P6 would more easily understand what she had to do to manage her illness and why if she understood enough about the treatment and her medical status. It was also noted in this example that, even if caregivers shared "everything" with their patients, they still tended to filter or translate information according to their perception of their child's ability to comprehend, considering their young age.

In addition to the child's right to know, other caregivers who shared everything believed patients would benefit from knowing. These caregivers tended to convey clear messages to the child about how to cope effectively with the treatment procedure and have transparent discussions about their health status, such as giving verbal encouragement to the child about positive results of a lab test. They also considered that patients' awareness about their health condition and status would be helpful to them eventually so that all of the family members could manage the patient's health more collaboratively. 
"Why not [telling the patient]? It's her body [P13], she's going through it, she needs to know. I mean, for me, I think it's dumb parents not telling their kids, "Oh, okay, you're sick." That's how you want to tell them? That they'll pass away? They don't know anything, they just know they are sick. But no, she knew she had cancer, she knew everything, that she could die from it. I think it's the right thing to do, is tell your child if they have something that's real serious, to tell them about it. Because if they tell ... Because sometimes you might forget that your kid's sick. And then like, "Mom, you know, I can't do that, I'm sick. I have cancer. I can't ... I don't have an immune system. I can't go outside." You know, if you want them to go outside and have fun, or if you're going somewhere to eat they remind you to put a mask on." - CG13 (Dad)

Here, the caregiver highlighted the complementary role of the patient's awareness of her illness. He described how it could be useful for parent-patient cooperative health management. He implied that for some situations (e.g., if the caregiver forgot and accidentally suggested activities that carry a health risk), a patient who was familiar with his or her health condition might be able to speak up about what they could and could not do. This could allow both caregiver and patient to be on the same page about how to manage the patient's health needs in daily life.

5.2.2 Minimizing Psychological Burden through Selective or Non-Disclosure. In contrast, some caregivers in our study selectively shared information with patients because they wanted to minimize the patients' psychological burden and distress. As parents, caregivers had already seen their child go through the pain and suffering from medical procedures and treatment. So, caregivers did not want their child to experience any more anxiety, stress, or sadness as a result of sharing distressing health information with them. Therefore, these caregivers tended to disclose only selective information to the patients, such as clinical visits for treatment and infection prevention requirements.

This selective disclosure also varied based on caregivers' preferences and decisions. Also, there were no instructions or guidelines given to caregivers about how and how much information they should share with the patients. Hence, the caregivers made efforts to decide how much information would be enough to enable the patients to cooperate with the treatment and not to have psychological distress. For example, CG12 commented that she decided to explain cancer as a general disease that it is just a "sickness" and did not tell P12 about the severity of cancer right after the diagnosis:

"I guess I was very concerned about letting him know that this could be fatal. I didn't think that would be helpful to him and that's why I shied away from the "cancer" word because so many people associate that and I thought eventually he's going to pick up on that. So, I just want to keep him, I guess in a way, safe from that idea." - CG12 (Mom)

In this case, CG12 believed the life-threatening aspect of cancer would negatively affect P12's mental well-being by causing fear. She thus purposely shared the illness-related information abstractly and vaguely with P12, explaining that he had a sickness in his blood instead of using the word "cancer" to hide the risks and mortality of the disease. In doing so, she reasoned about how much information and what type of information would be more appropriate to share. She further commented that she wanted the patient to "be a kid":

"I just wanted to let him be a kid as much as he could be, which he really couldn't be much during that year, but still didn't want to let him give that all up before he needed to" - CG12 (Mom)

This comment indicates CG12's honest intention for selective information disclosure to the patient. By emphasizing the word "kid," CG12 tried to differentiate "being a kid" and "being a cancer patient." CG12 implied that other healthy kids would not worry about their health condition or everyday activities because of fear of losing their health again; and she also considered that if P12 were to learn about his condition, he would consider himself to be a patient with a fatal disease 
and would have to relinquish what he used to do as a kid. So, to prevent P12 from having such pessimistic feelings, CG12 hid some information, even though she knew P12 would learn about it someday.

In extreme cases, some caregivers did not share any information about cancer with their child. This tendency was more salient among caregivers whose children were diagnosed at their very young age of 8 months - 3 years old. These caregivers intentionally did not create a record of the child's life as a patient so that the child would not know they had been ill in the past. For instance, one of the patients in our study, P5 (age 10 at the time of our interview), was initially diagnosed when he was just one year old. After the treatment and recovery period, he had a healthy life just like his peers. To keep his life normal, CG5 had not told him about cancer; consequently, even nine years after the treatment, P5 did not know that he was a cancer survivor. Instead, P5 was told and believed that his yearly check-ups for relapse were just general check-ups with his primary care provider. Similar to those caregivers who only shared selective information, CG5 did not want P5 to experience any psychological burden and distress from knowing about his history of cancer and the possibility of relapse. In particular, because of P5's very sensitive personality, CG5 regarded cancer as too heavy a topic to share and discuss with him. She also thought knowing about his cancer would cause him to struggle and feel different from his friends in a negative way.

"Because I don't want him to have that kind of burden, and feel like, 'I'm different from other kids.' So, because I told you he is sensitive, right. So, I don't want to tell him at this time. He would feel cancer is a very heavy topic for him, and still understand that." - CG5 (Mom)

Some of the caregivers who had not yet disclosed information about the illness to patients also worried about how and when they would eventually share illness-related information. These caregivers believed that the patients should ultimately know about their medical condition because they would need to manage their own body and health, but only when they would become more mature and comfortable enough to understand. To disclose health information, apart from telling the patients about what might happen (i.e. relapse), these caregivers would still have to explain what had already happened and to tell the patients that they were not normal. Also, the fact that the patients had been considering themselves as healthy people, just like others, made the caregivers' decision more complicated, despite knowing that an unexpected event such as relapse might come and break the peace. For example, CG5 shared her concern about disclosing the illness to P5 in the future.

"I think the most, for him, he didn't feel anything, and only the parents - we know. So there's a big challenge, for us, how to, for the past years, we don't feel, we feel lots of challenges, and we had lots of challenges, and it's all past. And for us, now, like I told you, we have the concern in the future. So how to, and also we have the challenge, how to teach him to those things.... And to prepare for the future. So, it's a big challenge for us, too. To tell him that he is a little bit different than other normal kids." CG5 (Mom)

CG5 knew that she had to tell P5 in the future so that he could prepare for the risk of a relapse. However, she was concerned that telling P5 everything would reveal to P5 that he was different from other healthy children. Here, the caregiver showed her wish to avoid a situation where the child might feel different and disconnected from his peers or the world around him, which could further cause a negative feeling of separation, such as anxiety or depression. Considering P5's sensitive personality, CG5 was extra cautious about any potential impact on P5's personality and tried to prevent $\mathrm{P} 5$ from figuring it out accidentally.

5.2.3 Consequence of non-disclosure. In the study, we found that non-disclosure of the illness became another burden for the caregivers to carry. When caregivers hid information from the patients, it sometimes prevented caregivers from seeking or receiving support from others. Because 
caregivers did not want any psychological burden on the patient, they had to completely hide their own struggles from the patients by not talking about any subject related to illness or showing their concerns. For example, CG12 experienced loneliness and isolation due to a lack of social support; she did not want P12 to overhear her talking to others about the illness accidentally.

"Probably the isolation... the complete and total isolation at a time when you really need to talk maybe, but there's just not an opportunity for me. I don't know if other families have the same experience. They must, but just from what I've seen, many other families have these conversations in front of their kids and I just don't think that it's the right thing to do for us, at least. So that makes it very hard that first year, now that he's in school, I have some time to myself." - CG12 (Mom)

CG12 had to refrain from talking about disease in front of P12, and later on, she realized that this restriction isolated her from seeking and obtaining necessary social support. This compounded other barriers to social support. After transitioning home from the hospital, CG12 realized she had lost the social support she used to have from care team members during P12's hospital stay. Moreover, in the early stages of treatment, CG12 had to provide attentive care and did not have time to seek support for herself. Her husband was her only outlet, but he was also busy with his job. Thus, for years CG12 did not have opportunities to share her struggles and receive the support she needed.

In this section, we found a spectrum of information disclosure behaviors of caregivers, from full disclosure to non-disclosure. We also identified different preferences and rationales regarding how caregivers made decisions about information disclosure. Some believed patients had a right to know, some believed sharing this information would be useful for the patient's self-management and collaborative care, and others believed sharing this information would be psychologically harmful, which might impact patients' mental development. We also found that some caregivers experienced an unexpected burden associated with partial or non-disclosure, despite patients having less distress about their medical status. In addition, caregiver participants were not confident about when, where and how much information needed to be shared with patients.

\subsection{Tension between facilitating cooperation for treatment and maintaining discipline}

Lastly, a tension arose between encouraging the patient's cooperation with treatment and the discipline that caregivers had built within the family. Because of the regular clinic visits involved intensive treatments, patients constantly received the most careful attention and assistance from everyone surrounding them, including hospital staff and extended family members. However, this became a disruption to caregivers' abilities to keep and maintain discipline at home when patients asked for more than what they needed or became too reliant on others. While much attention and encouragement promote better patient cooperation, caregivers were concerned with its long-term impact on their children, such as not being disciplined and independent. Caregivers felt that it was challenging to balance generosity and their original boundaries for discipline.

Though it varied depending on treatment stages and the patient's condition, all of the patient participants were required to visit clinics somewhat regularly. After leaving the hospital, patients were supposed to visit a clinic twice per week at first, and then once per week until the "100-day" milepost, which indicated the end of the critical period for managing complications. Later on, clinic visits were typically scheduled every month for six months, every two months for the next six months, and every three months for the following year. Clinic visits included lab tests, clinical consultations, physical checkups, and also might consist of infusions or other relevant procedures based on lab test results. Caregivers thought these visits presented major disruptions to the patients' daily routines. Sometimes the visits prompted patients to realize they were still sick, and the treatment itself was painful for the young patients (e.g., pokes, infusions). Other times the visits 
required canceling patients' routine activities, such as missing a school day, and patients often had to adjust to treatment routines afterward (e.g., might return to the clinic for another infusion).

To minimize patients' distress from these clinic visits and expedite the patient's cooperation with painful treatment, caregivers tried their best to proactively accommodate the patients' needs in coordination with care team members. Caregivers mentioned several strategies they implemented. For instance, all patients in the study received new toys to complete a clinic visit successfully or negotiated what they wanted to have after upcoming clinic visits. Nearly all of the patients received extended screen time to be distracted from a lengthy painful treatment procedure. Many received cards, stickers, and other free gifts from care team members to make them feel happy. As such, all of them received attention and assistance throughout those clinic visits or short hospital stays.

The focused attention and rewards motivated patients to better prepare for clinic visits and to cooperate with painful treatments. CG8 mentioned that she developed a reward system to encourage P8 to better cooperate with "the poke," which is the most painful medical procedure that is used either to inject liquid substances directly in to a vein for treatment or to remove fluid surrounding the brain and spinal cord for examination through a tube inserted into the chest:

"Well, I think he's very reward-driven and so I've found that the toys are a big motivation, so being able to go to the store and pick out a poke prize helps him forget about [the pain] ... I mean, it seems to work. He does not want to go sometimes. There was one time he had these seven pokes in one day, and so... Well, just like, think, you get more, more and bigger poke prize.” - CG8 (Mom)

This quote shows that CG8 actively utilized post-treatment rewards as a coping strategy to motivate P8 to go to the clinic and to distract him from the pain of the treatment process. CG8 even offered $\mathrm{P} 8$ a bigger prize if the treatment was more painful than usual, reasoning that it was very imperative to get the treatment done successfully.

However, while caregivers thought that they needed to provide the patients some consolation for physical pain and psychological distress, they worried that using rewards might conflict with their parenting style. Except for CG14, all of the caregiver participants had more than one child and they had built and maintained discipline with their children. But, in order to cope with the sick child's unique situation, they had to change or deviate from their rules. Several caregivers expressed concern about behaviors they had noticed, such as their child becoming demanding and asking for more than they needed, or not being satisfied with what they received. For instance, CG8 said that P8 had become spoiled by overusing chances to get rewards:

"He is spoiled, admittedly. From both sides. Because he ... well, because we give him poke prize so he gets toys. He has lots of toys because when he was in the hospital, everyone donates and gives lots of presents. So he's got more toys than anyone that I know." - CG8 (Mom)

The caregivers also made exceptions to their disciplinary approach by letting the patients do things during clinic visits that they had not allowed otherwise. For example, CG12 explained why she let $\mathrm{P} 12$ have unlimited screen time at the hospital when she would never allow this at home:

"He [P12] is allowed screen time. We definitely try to limit it, but this kind of situation is hard. He has his own tablet. He and his brother are allowed to watch certain YouTube channels and certain apps. During wait times, he's usually with a screen of some sorts, because he's not allowed to do it at home. So he takes full advantage." - CG12 (Mom)

Like CG12 in the quote above, who eased house rules to reduce her child's distress from visiting the hospital, many caregivers felt that they could not restrict patients' behavior in the clinic. During clinic visits and other distressing treatments, parents perceived that they did not have other options besides deviating from house rules and allowing extra screen time.

However, at the same time, most caregivers worried about the cognitive effects of rewards on patients. Some reported that patients expected the same level of attention and assistance they had received during long stays at the hospital after they had returned home. During the hospital 
stay, to reduce the patients' anxiety and encourage cooperation, clinicians, family caregivers, and other hospital staff attended very closely to the patients and provided whatever they wanted. This made patients get used to such close attention and tend to expect the same level of attention upon returning home. This tendency was salient among patients who were diagnosed at a young age and had lived in the hospital for relatively longer time periods. For example, CG2 described how P2 had changed and became spoiled and overindulged by parents and other adults after his long hospital stay:

"He is also being brought up in the cancer world, he is a little bit hand fed. You know.. little almost spoiled.. I must say he understands and expects when the nurse comes in, they are gonna say- what can I get you? What can I do for you? Let me help you.. you know.. So we deal with it a little bit at home. (...) Yeah.. he will pitch in with chores and things but quick to say.. ah.. I cant! I will take a nap and [P2' brother] can do it. Whereas it is hard not to compare." - CG2 (Mom)

Here, CG2 noticed the difference in P2 over time since he had spent a long time in the hospital, surrounded by people pampering him. Because of this vast amount of attention and assistance P2 was accustomed to getting all the time, the caregiver recognized that his attributes had changed, such as lack independence and expecting others to do things for him, even for little chores at home. CG2 further commented that the lasting effect on P2 also would affect his siblings since P2 always wanted more attention and possessions than other siblings at home.

Similarly, in the case of P7, after her transplant, CG7 observed that P7 tried to take advantage of being sick to get more attention from others:

"Just kind of reinforcing that we're in a different setting now. Things are different now. We have to share with our brother. It's not nice to lie about having a stomachache or a headache and things like that to get medical attention like she was used to, which meant attention from mom or providers or things like that." - CG7 (Mom)

This example demonstrates CG7's desire for P7 to return to desirable behaviors after receiving more attention and care during the transplant period. CG7 noticed that after coming back home from the hospital, P7 sometimes lied about her symptoms to get greater attention, and she worked harder to explain to P7 about the change in situation (transitioning to home from the hospital) and to reestablish house rules shared with other family members. Particularly with P7's two other siblings at home, CG7 struggled to keep the balance between her effort to provide attentive medical care for P7 and her desire to maintain discipline for all of her children.

In this section, we explored the provision of rewards, assistance, and attention to reduce patients' psychological distress and encourage better cooperation during clinical visits, and we demonstrated caregivers' concerns about disrupting their disciplinary approach. We also discussed unexpected consequences upon returning home from the hospital, such as patients continuing to demand lots of attention and assistance by lacking independence and affecting their siblings by disrupting discipline at home. We found that caregivers of pediatric patients struggled to balance their disciplinary expectations as parents with their caregiving responsibilities of minimizing the patients' distress during clinic visits and treatment.

\section{DISCUSSION}

Our findings revealed the dilemmas caregivers faced when encountering three types of tensions between parenting and caregiving responsibilities: tension between maintaining physical health and meeting social needs, tension between disclosing health-related information and minimizing the psychological burden on patients, and tension between facilitating treatment cooperation and maintaining discipline. This care work is not solitary; it involves communication and coordination with others. First, caregiving work for pediatric patients is a process of managing the patient's illness and health conditions on behalf of the patient, including translating knowledge between clinicians 
and the child and transferring or sharing the child's health information with others. Second, as the findings show, as parental caregivers (often both parents) work through tensions between parenting and caregiving, they renegotiate their relationships with others, including the child patient, other family members, healthcare providers, and teachers. In this sense, caregiving work for pediatric patients can be viewed as collaboration among parental caregivers, family members, and others who are involved in the pediatric patient's life. Below we elaborate on two processes we regard as critical to the collaborative caregiving endeavor. Specifically, we discuss the process of assessment and re-assessment that parental caregivers employ to balance tensions between parenting and caregiving responsibilities by drawing on a theoretical framework of "parenthood stages." We then discuss parental caregivers' ongoing efforts to work with uncertainty across time scales in pediatric care management.

\subsection{Balancing tensions as a process of assessing and re-assessing}

Based on our analysis of the tensions between parenting and caregiving, we reveal that instead of transitioning from a parent role to a caregiver role [15], parental caregivers experience and grapple with both responsibilities together in an ongoing process. Similar to Miller et al. [28], who showed parental caregivers perceiving, shifting, and taking on different caregiving roles over time through negotiating with the patient during inpatient care (e.g., the roles of assistant or navigator played at the beginning of a hospital stay), our caregivers attempt to fulfill two types of responsibilities depending on the circumstances. Tensions occur when caregivers are expected to carry out both caregiving and parenting tasks simultaneously. To balance the tensions, parental caregivers in our study go through an ongoing process of assessing, acting, and re-assessing. In this section, to deepen our account of this ongoing process, we draw on prior work from $\mathrm{CSCW}$ and theory from psychology.

We distinguish two types of responsibilities that parental caregivers consider: 'caregiving' and 'parenting.' Previous works in CSCW have examined how different types of caregivers perceive and carry out caregiving roles $[6,15,28]$. These studies describe different responsibilities and challenges of caregiving, such as playing multiple roles during inpatient care [28], balancing personal tasks and caregiving obligations [6], and managing information and emotional work during the transition to becoming a caregiver [15]. Aligning with these studies, our findings reveal caregivers' struggle to fulfill their caregiving responsibilities (e.g., preventing infection, disclosing information, facilitating patient cooperation) while carrying out their parenting responsibilities. After the discharge from the hospital to the home, caregivers have to keep fulfilling caregiving responsibilities alongside parenting responsibilities, causing the tensions we identified.

For a better understanding of how parenting responsibilities shift over time, we draw on Galinsky's [10] six stages of parenthood, a theory of parenthood development often used in the fields of psychology, education, and communication. This framework describes how parents perceive their experience as parents in relation to six distinct stages of child development: image-making (pregnancy), nurturing (birth-age 2), authority (age 2-5), interpretative (age 6-12), interdependent (age 13-19), and departure (above 19). Most of our participants were in the authority stage or the interpretative stage when they adopted new caregiving responsibilities. During the authority stage, parents mainly work on establishing their identity as parents by setting up rules at home. In the interpretative stage, parents work on interpreting the world (e.g., facts, skills, experiences, etc.) to their children and setting standards for them. Parents also make decisions about how to behave toward their children and how to interpret themselves to their children.

As children develop their own expectations of the world, including what parents should do, parents reflect on and evaluate their past identity as a parent (typically constructed in the authority stage). This self-evaluation process involves making decisions consciously or unconsciously

Proc. ACM Hum.-Comput. Interact., Vol. 3, No. CSCW, Article 153. Publication date: November 2019. 
(e.g., how much they should be involved in their children's lives) as parents strive toward their interpretation of what it means to be a "good" parent.

Turning back to our findings, Galinsky's theoretical framework helps us understand the struggles of our caregivers from the parental point of view during the interpretative stage. For example, in our study a mom caregiver (CG12) tried to meet her own standard of a good parent (the desired self) by letting P12 interact with his friends even when one friend had a cold, as she believed that it was a parenting responsibility to help develop the child's social skills and needs. Some caregivers - particularly those whose children were diagnosed at a very young age of one or two years might have different standards for being a good parent compared to parental caregivers whose children were diagnosed at older ages. Despite such differences in standards, they also experience similar tensions between caregiving and parenting needs. For instance, as shown in the findings, CG5 had to adjust to caregiving responsibilities when her one-year-old child was diagnosed. This early adoption of new identity as a caregiver allowed her to set all the rules in relation to health management in the authority stage [10]. While keeping her child healthy, she believed that she was fulfilling her standards as a good parent by not disclosing the illness to the child. However, she later recognized the necessity of information disclosure for better treatment and health outcomes. Thus, she faced a tension between meeting caregiving needs (telling P5 everything about illness) and being a good parent (minimizing the psychological burden on the child).

Although we can distinguish caregiving and parenting responsibilities analytically, in practice parental caregivers do not encounter either responsibility independently of each other; they are intricately intertwined. Over time, parental caregivers engage in an ongoing process of weighing caregiving responsibilities against parenting responsibilities. The caregivers continually reassess their actions relative to shifting parenting and caregiving needs. Since children continue to develop, parents cannot settle on any given balance between parenting and caregiving responsibilities. Parents' approaches to parenting and their relationship to their children will continue to shift[10], and the children's illness may improve and/or worsen. Thus, balancing these tensions is never complete but an ongoing process.

Caregiving responsibilities force parental caregivers to reinterpret themselves and complicate their imagined, desired, and expected role as a good parent. A mismatch between their desired role and the actual role causes parental caregivers to feel disturbed. For example, CG3 believed a good parent would teach hugging as a way to show affection and encouraged P3 to hug. As a parent, she was happy to see how P3 was becoming a lovable and affectionate boy. However, after the BMT procedure, CG3 reinterpreted her job as a caregiver for her child and realized the necessity of stopping him from being affectionate to others while also maintaining his social, friendly personality growth. Since suddenly forbidding P3 from being social with his friends felt unreasonable and contradicted CG3's expectations for a good parent, CG3 and her husband had to constantly assess and reassess their actions to balance those two needs in everyday care practice.

Unlike parents without caregiving responsibilities, parental caregivers have to set flexible standards - sometimes even conflicting standards - and need more control over interpreting the world to their child patients. This ongoing process of assessment and re-assessment to balance different needs, expectations, and responsibilities created a unique challenge for parental caregivers in the study.

\subsection{Working with uncertainty across time scales}

Dealing with tensions between caregiving and parenting responsibilities, our caregivers continually made efforts to balance these responsibilities over time. They often weighed immediate consequences that had greater certainty against potential, long-term consequences that had less certainty. For instance, efforts to address immediate concerns included isolating the child to minimize the 
possibility of infection, withholding information to avoid psychological burden on the patient, and providing rewards to ensure cooperation with treatment. On the other hand, supporting the child's social development, cultivating the child's ability to self-manage illness, and avoiding dependence on rewards are long-term concerns with uncertain consequences. For parents, addressing concrete problems in the short-term could potentially jeopardize long-term outcomes; enduring some immediate consequences might lower the probability of long-term consequences. Thus, parents had to consider and anticipate whether the risk and severity of immediate consequences for the patient's health were prohibitively high (e.g., life-threatening infection, emotional scarring).

As an example of this, consider our finding regarding the caregivers' responsibilities to translate health-related information to be workable within the routines and demands of the patient's daily life. After the BMT, as the parent and child transitioned out of the hospital and into the home, they were faced with new questions about the conditions under which it was safe for the child to come into physical contact with friends and relatives. This caregiver's translation role resonates with prior work in CSCW. Kaziunas et al.[14] described how patients with chronic illness living in Flint, Michigan translated their doctors' orders to fit within the social, localized context and lived experience of daily life. Just as it was not workable for those patients to adopt their doctors' orders without modification, in our study it was not workable for parental caregivers to completely isolate their child from social contact in their everyday life. Doing so would stunt the patient's social development, and the caregiver would fail to fulfill the patient's social needs over the long term.

Our findings also resonate with recent work by Nunes and Fitzpatrick [32] and prior works by Annemarie Mol $[29,30]$ on "persistent tinkering" involved in managing illness. This line of work has called attention to how patients and caregivers engage in an ongoing process of mundane adjustments in daily life, such as keeping medications in a particular place or assessing whether to take medication on schedule or to adopt an irregular schedule to control symptoms at particular times of the day or week [32]. Patients and caregivers interpreted and implemented these activities in order to overcome the mundane challenges of living with the disease, such as cognitive and physical limitations.

Compared with these prior works, for our parental caregivers, the stakes were higher, the time scales were broader, and the consequences of tinkering were less certain. Similar to "persistent tinkering," parental caregivers in our study engaged in an ongoing process of acting and evaluating actions (see Section 6.1). While previous studies focused on adjustment in daily activities, which mostly considered immediate consequences, we extend this concept of persistent tinkering by emphasizing the different time scales our parental caregivers considered as they balanced tensions between parenting and caregiving. For the persistent tinkering, they engaged in day-to-day adjustment as well as re-adjustment for the long-term benefit of the child's social and emotional growth. Parental caregivers did not always have the advantage of trying something out, observing how well it worked, and adapting to ongoing care work accordingly. Some of the consequences of experimenting could be immediate (e.g., emotional distress from sharing) and severe (e.g., life-threatening infection); and other consequences were uncertain and developed over months and years (e.g., the child's social development, or the child's expectation of rewards). The latter consequences, in particular, were combined with other long-term factors, such as personal, social and cultural, or educational development. These were usually invisible initially, and uncertain and unpredictable until later.

Because of this, compared with some of the tinkering afforded to other patients and caregivers, parental caregivers were required to make greater efforts in their ongoing tinkering by carefully observing, monitoring any unintended changes in their children over time, and trying to think ahead about proximal consequences. This challenge was demonstrated in the example of Section 5.2. The

Proc. ACM Hum.-Comput. Interact., Vol. 3, No. CSCW, Article 153. Publication date: November 2019. 
caregivers who did not disclose information about illness were waiting until their children became more mature to manage the psychological burdens, but caregivers had difficulty with figuring out how and when to start handling this long-term impact appropriately. Thus, a salient aspect of the care work performed by parental caregivers in this study was grappling with situations in which short-term exigencies clash with long-term matters of importance, and in which responsibilities associated with parenting clash with responsibilities associated with caregiving. This illustrates the importance of parents anticipating potential consequences for their children across short- and long-term time scales.

\section{DESIGN IMPLICATIONS}

In this section, we detail design implications based on the study findings. We argue that systems should not merely address what caregivers can do for patients or what the patient needs, but should also include how to address caregivers' other conflicting responsibilities and needs as parents raising a young child with demanding medical needs.

\subsection{Integrating caregiving and parenting as a collective endeavor}

As discussed in Section 6.1, parental caregivers engage in an ongoing process of interpretation, assessment, action, and re-assessment to negotiate between conflicting caregiving and parenting needs. In this process, caregivers do not address these two needs separately but struggle to balance them simultaneously. Meeting caregiving needs may allow them to feel successful as caregivers but poor as parents or vice versa. To help ease the tension and balance the two roles, we suggest that these two types of needs should be connected and worked on together so that they can inform each other. Instead of being held and addressed in parallel, both needs should create a loop that can be evaluated coherently. In this loop, each need can encourage caregivers to better reflect on the other one. For instance, when caregivers meet caregiving needs and consider themselves good caregivers, they can also reflect on their parenting role and try to reach an acceptable balance between caregiving and parenting.

Our study has shown that parental caregivers try to seek or utilize feedback from other expert individuals, particularly when gauging social development of their child or looking for some solution in a tricky situation in between parenting and caregiving. Since these experts, such as a physician, a nurse, or a school teacher, have different expertise and knowledge about the child's needs, necessary resources, and coping solutions, parental caregivers value and use their feedback to make or confirm their own assessment when balancing their dual needs and responsibilities of caregiving and parenting. Specifically, while caregivers evaluate their parenting needs based on teachers' feedback (as parents), caregivers measure the achievement of their caregiving work based on the providers' report on the patients' health (as caregivers). However, because they may receive distinct (or even conflicting) inputs from different resources that do not talk to one another, the feedback received can represent only partial views. These partial views may conflict. When a conflict occurs in those external evaluations and interpretations, it can fall to the parental caregivers to reconcile them in their course of action, which can potentially even aggregate their struggle. Thus, it is necessary to collect, organize, compare, and integrate the feedback from different stakeholders with distinct, partial perspectives on the child's health and development.

Our findings suggest an opportunity for creating a shared information space that can support this new approach to managing the two needs. An example of this intervention can be a sociotechnical platform where caregivers collectively track and record logs about the pediatric patient with other key stakeholders, such as a physician, a nurse, a teacher, and other important family caregivers (spouse or grandparent). Each stakeholder can track different aspects of the patient: for instance, a teacher at school can contribute observations about key concerns, flags, or achievements involved 
in the patient's social development and specific activities done in classes. For the child's privacy, we consider that the permitted key stakeholders above would have access to the system, but that caregivers could still manage to share the logs only with selected stakeholders on behalf of the child. Tracked logs could be shared among stakeholders so that they can review and make necessary comments on each log. This platform could allow caregivers to share specific situations where caregivers have particular difficulty (tension) in balancing caregiving and parenting responsibilities while learning how to handle similar situations in the future.

By facilitating shared communication among stakeholders that incorporates different viewpoints and feedback, an integrated system could provide caregivers the opportunity to connect caregiving and parenting needs and to reflect on and evaluate their actions. We do not claim that such a system will resolve all conflicts since some stakeholders may place greater importance on health and safety. However, we believe that this will still provide a chance to open up a new conversation among the care network by allowing multiple perspectives to be represented together regarding the child's current situation. Such a system may be able to strengthen the parental caregiver's self-reflection and assessment process and also help to create common ground and awareness among stakeholders about the child's health and safety as well as social development, which could assist parental caregivers in balancing their responsibilities.

\subsection{Combining illness and child development trajectories}

To better understand the struggles caregivers experience in the course of their child's disease management, we look at the concept of "illness trajectory" by Strauss et al. [36]. They describe an illness trajectory as involving "not only the physiological unfolding of a patient's disease but the total organization of work done over that course [of illness], plus the impact on those involved with that work and its organization" [38]. In detailing the types of work involved in the illness trajectory, Strauss et al. also note that patients and caregivers try to balance illness work (i.e. illness-related work, such as safety work and comfort work) with daily life work (such as child rearing or marital work) in order to maintain a relative equilibrium [36]. Changes in illness or everyday life can affect one another, at times requiring efforts to switch between different types of work. In our study, we saw that balancing caregiving and parenting work is a specific type of challenge for caregivers, where they could benefit from further support

Upon the pediatric patient's diagnosis, when parents become caregivers of a child with cancer, they are provided with information about the expected treatment trajectory. For instance, there are guidelines for parents of children with cancer issued by the National Cancer Institute [1]. They provide an overall trajectory of treatment, instructions for children's health management, and coping strategies for emotional distress. However, these guidelines do not address the challenges parental caregivers may encounter when they face tensions between caregiving and parenting responsibilities. This is because primarily caregiving work that addresses the patient's medical needs is considered in existing pediatric care practices [17], overlooking caregivers' responsibility for simultaneously managing important aspects of the child patient's development and associated needs, such as their social and educational growth. Hence, this lack of integration is an area in which the system falls short of a more patient-centric approach to care and makes it difficult for caregivers to expect, anticipate and prepare for such challenges with two different set of responsibilities. Thus, we see an opportunity for the design of products and services to integrate the treatment trajectory with the child development trajectory to better support caregivers in managing the illness trajectory. Considering the two trajectories together in a more integrated way could allow caregivers to more readily anticipate and reason about their responsibilities because they could plan for and observe their children's status as patients and as kids at the same time. 
To our knowledge, there are very few existing technical interventions to support caregivers in their caregiving or parenting work $[9,19,38]$. These systems exist for managing either trajectory independently. For example, "BMT Roadmap" is a web-based application recently designed for parental caregivers, which integrates patient-specific information for bone marrow transplant patients during the treatment process [9,34]. Along with clinical reports and medication information, this application provides the trajectory of patient treatment. One of its main functions is the visual representation of the treatment trajectory, which provides detailed descriptions and relevant information for each phase of the treatment as a patient goes through the inpatient hospitalization. The findings from that work show that the visual representation of the BMT Roadmap helps caregivers know what to expect and look for during inpatient treatment, which in turn helps to reduce caregiver burdens. On the other hand, research by Suh [37, 38], Kientz[19] and colleagues provides examples of different technologies (Baby Steps, @BabySteps, Baby Steps Text) for supporting parents in tracking their children's developmental milestones. For instance, Baby Steps is a software that encourages parents to record information about their child's development and review the data frequently. Based on an existing developmental screening survey, the software provides parents a list of questionnaires based on six categories of age-appropriate milestones (Communication, Fine Motor, Gross Motor, Problem Solving, Personal-Social, and Overall). The system offers various functions like uploading pictures or videos of the child accomplishing goals, adding notes, and displaying color-coded icons to help parents explore the milestone list easily.

Based on our findings, we see value in integrating functionality from such systems to support caregiving and parenting work. An integrated system could provide caregivers with useful references to gauge their child's treatment as well as developmental condition, assisting them in anticipating needs as well as adjusting their actions. For instance, the system could suggest specific topics about self-management that is necessary for the child to learn based on his/her current treatment stage and offer guidance for proper medical information disclosure and how to deliver that information in an age-appropriate way. Specifically, in the case of BMT populations, by integrating important milestones in the treatment trajectory (e.g., Day 100 after transplant) and child development trajectory (e.g., communication milestones for a 6-year-old child and starting kindergarten), the system could guide caregivers to identify what information to provide to the child, how to communicate it, and what conflicts they might expect from managing the child's health and social needs. While this is beyond the scope of our work in this paper, ideally such a system should also allow caregivers to tailor it based on their own situations, considering their family culture, parenting style, home discipline, school culture, and the individual child's personality and development level.

As implied so far, beyond simply combining two trajectories, an integrated system would need to incorporate how different milestones may relate to or impact one another. For example, the system could provide a timely notice or flag for potential tensions in advance (e.g., infection prevention and social interaction), but also importantly afford a chance for caregivers to think about and discuss potential challenges and their decisions about how to approach these. This may also open up a valuable opportunity to bring experts into the discussion, as discussed earlier, to reason about how to make better decisions when caregivers experience a tension between conflicting responsibilities. While such a system may not directly or completely resolve a tension, it could help the caregiver to be better prepared and seek advice or support from others in a timely manner. To our knowledge, this kind of support is currently not available through existing technologies. If the system allows caregivers to record instances where a tension occurs along the illness trajectory, it could also help them to reflect on their decisions. Based on such records of specific incidences, an expanded system could offer a way to learn from others who have gone through similar trajectories - either treatment or development, or combined - by sharing with other parental caregivers who might 
have struggled with similar tensions, thereby facilitating a form of social support. In summary, we believe an integrated system has the potential to aid caregivers in organizing and balancing the work related to parenting and caregiving in the illness trajectory of the child patient.

\section{LIMITATIONS}

There are some limitations to this study. Given its qualitative nature, the findings are specific to the particular context that we examined. We focused on caregivers of children (age 6-12) with blood cancer. This kind of chronic illness involves specific needs, such as infection prevention due to a weakened immune system and disclosure of sensitive information about illness and treatment (e.g., life-threatening characteristics of the illness). Since this study addresses tensions related to these characteristics (e.g., infection prevention and social needs), further research is necessary to explore if and how caregivers in other chronic illness contexts experience tensions in balancing parental and caregiving needs. Similarly, the study focuses on a specific group of caregivers. Our study included caregivers of children 6-12 years old who receive treatment at a large teaching hospital in the U.S. Midwest. Experiences may differ in other settings and populations (e.g. with caregivers of teens). However, we believe that conflicts between parenting and caregiving needs may arise in other chronic illness contexts (and may be similar or different from tensions observed in this study) and warrant further examination.

\section{CONCLUSION}

In this study, we examined the tensions that parental caregivers faced in carrying out both caregiving and parenting needs and responsibilities, and their struggles to balance those two, sometimes conflicting, roles. We believe this work makes two important contributions to the CSCW community. First, we demonstrate how specific tasks of caregiving and parenting can have conflicting priorities. Second, we reveal that caregiving and parenting roles are separate, but virtually inseparable in practice. Extending on prior work about dilemmas of parental caregivers, our findings suggest implications for what responsibilities or needs researchers and designers should consider for developing sociotechnical systems to support the caregivers of pediatric patients and how the systems can support caregivers to better balance their two sets of responsibilities in their pediatric care.

\section{REFERENCES}

[1] [n. d.]. Comprehensive Cancer Information. http://www.cancer.gov/

[2] Tawfiq Ammari and Sarita Schoenebeck. 2015. Networked Empowerment on Facebook Groups for Parents of Children with Special Needs. In Proceedings of the 33rd Annual ACM Conference on Human Factors in Computing Systems (CHI '15). ACM, New York, NY, USA, 2805-2814. https://doi.org/10.1145/2702123.2702324

[3] Andrew B. L. Berry, Catherine Lim, Andrea L. Hartzler, Tad Hirsch, Edward H. Wagner, Evette Ludman, and James D. Ralston. 2017. How Values Shape Collaboration Between Patients with Multiple Chronic Conditions and Spousal Caregivers. In Proceedings of the 2017 CHI Conference on Human Factors in Computing Systems (CHI '17). ACM, New York, NY, USA, 5257-5270. https://doi.org/10.1145/3025453.3025923

[4] Hugh Beyer and Karen Holtzblatt. 2000. Contextual design defining customer-centered systems. Morgan Kaufmann.

[5] Claus Bossen, Lars Rune Christensen, Erik Grönvall, and Lasse Steenbock Vestergaard. 2013. CareCoor: Augmenting the coordination of cooperative home care work. International journal of medical informatics 82, 5 (2013), e189-e199.

[6] Yunan Chen, Victor Ngo, and Sun Young Park. 2013. Caring for caregivers: designing for integrality. In Proceedings of the 2013 conference on Computer supported cooperative work. ACM, 91-102.

[7] Christine Eiser, J Richard Eiser, and Veronica Greco. 2002. Parenting a child with cancer: Promotion and preventionfocused parenting. Pediatric rehabilitation 5, 4 (2002), 215-221.

[8] Shelly Farnham, Lili Cheng, Linda Stone, Melora Zaner-Godsey, Christopher Hibbeln, Karen Syrjala, Ann Marie Clark, and Janet Abrams. 2002. HutchWorld: Clinical Study of Computer-mediated Social Support for Cancer Patients and Their Caregivers. In Proceedings of the SIGCHI Conference on Human Factors in Computing Systems (CHI '02). ACM, New York, NY, USA, 375-382. https://doi.org/10.1145/503376.503444 
[9] Alex J Fauer, Flora Hoodin, Leah Lalonde, Josh Errickson, Lyndsey Runaas, Tracey Churay, Sajjad Seyedsalehi, Casiana Warfield, Grant Chappell, Kristina Brookshire, et al. 2018. Impact of a health information technology tool addressing information needs of caregivers of adult and pediatric hematopoietic stem cell transplantation patients. Supportive Care in Cancer (2018), 1-10.

[10] Ellen Galinsky. 1982. Between generations: the six stages of parenthood. Berkley Books.

[11] Amelia Gibson and Samantha J. Kaplan. 2015. "A Family Like Ours": Demographic Variations in Information Seeking Behavior and Community Participation Among Parents of Individuals with Autism Spectrum Disorder (ASD). In Proceedings of the 78th ASIS\&T Annual Meeting: Information Science with Impact: Research in and for the Community (ASIST '15). American Society for Information Science, Silver Springs, MD, USA, Article 60, 4 pages. http://dl.acm.org/ citation.cfm?id=2857070.2857130

[12] Barbara L. Jones. 2012. The Challenge of Quality Care for Family Caregivers in Pediatric Cancer Care. Seminars in Oncology Nursing 28, 4 (2012), 213-220. https://doi.org/10.1016/j.soncn.2012.09.003

[13] Elizabeth Kaziunas, Mark S. Ackerman, Silvia Lindtner, and Joyce M. Lee. 2017. Caring Through Data: Attending to the Social and Emotional Experiences of Health Datafication. In Proceedings of the 2017 ACM Conference on Computer Supported Cooperative Work and Social Computing (CSCW '17). ACM, New York, NY, USA, 2260-2272. https://doi.org/10.1145/2998181.2998303

[14] Elizabeth Kaziunas, Mark S. Ackerman, and Tiffany C. E. Veinot. 2013. Localizing Chronic Disease Management: Information Work and Health Translations. In Proceedings of the 76th ASIS\&T Annual Meeting: Beyond the Cloud: Rethinking Information Boundaries (ASIST '13). American Society for Information Science, Silver Springs, MD, USA Article 11, 10 pages. http://dl.acm.org/citation.cfm?id=2655780.2655791

[15] Elizabeth Kaziunas, Ayse G Buyuktur, Jasmine Jones, Sung W Choi, David A Hanauer, and Mark S Ackerman. 2015 Transition and reflection in the use of health information: the case of pediatric bone marrow transplant caregivers. In Proceedings of the 18th ACM Conference on Computer Supported Cooperative Work \& Social Computing. ACM, 1763-1774.

[16] Laura MJ Kerr, Margaret B Harrison, Jennifer Medves, Joan E Tranmer, and Margaret I Fitch. 2007. Understanding the supportive care needs of parents of children with cancer: An approach to local needs assessment. Fournal of Pediatric Oncology Nursing 24, 5 (2007), 279-293.

[17] Gail M Kieckhefer and Cristine M Trahms. 2000. Supporting development of children with chronic conditions: From compliance toward shared management. Pediatric nursing 26, 4 (2000), 354.

[18] Julie A. Kientz. 2010. Understanding Parent-pediatrician Interactions for the Design of Health Technologies. In Proceedings of the 1st ACM International Health Informatics Symposium (IHI '10). ACM, New York, NY, USA, 230-239. https://doi.org/10.1145/1882992.1883025

[19] Julie A Kientz, Rosa I Arriaga, and Gregory D Abowd. 2009. Baby steps: evaluation of a system to support recordkeeping for parents of young children. In Proceedings of the SIGCHI Conference on Human Factors in Computing Systems. ACM, 1713-1722.

[20] Charalampos Kyfonidis and Marilyn Lennon. 2016. Mummy, Why Do I Have Diabetes?: A Tangible Interface For Educating Children With Type-1 Diabetes. In Proceedings of the The 15th International Conference on Interaction Design and Children (IDC '16). ACM, New York, NY, USA, 524-528. https://doi.org/10.1145/2930674.2935979

[21] Susan A. LaValley, Elizabeth A. Gage-Bouchard, Michelle Mollica, and Lynda Beaupin. 2015. Examining Social Media Use Among Parents of Children with Cancer. In Proceedings of the 78th ASIS\&T Annual Meeting: Information Science with Impact: Research in and for the Community (ASIST '15). American Society for Information Science, Silver Springs, MD, USA, Article 89, 3 pages. http://dl.acm.org/citation.cfm?id=2857070.2857159

[22] Leslie S. Liu, Sen H. Hirano, Monica Tentori, Karen G. Cheng, Sheba George, Sun Young Park, and Gillian R. Hayes. 2011. Improving Communication and Social Support for Caregivers of High-risk Infants Through Mobile Technologies. In Proceedings of the ACM 2011 Conference on Computer Supported Cooperative Work (CSCW'11). ACM, New York, NY, USA, 475-484. https://doi.org/10.1145/1958824.1958897

[23] Leslie S. Liu, Kori M. Inkpen, and Wanda Pratt. 2015. "I’m Not Like My Friends": Understanding How Children with a Chronic Illness Use Technology to Maintain Normalcy. In Proceedings of the 18th ACM Conference on Computer Supported Cooperative Work \&\#38; Social Computing (CSCW '15). ACM, New York, NY, USA, 1527-1539. https: //doi.org/10.1145/2675133.2675201

[24] Kiel Long, Lyndsey L. Bakewell, Roisin C. McNaney, Konstantina Vasileiou, Mark Atkinson, Manuela Barreto, Julie Barnett, Michael Wilson, Shaun Lawson, and John Vines. 2017. Connecting Those That Care: Designing for Transitioning, Talking, Belonging and Escaping. In Proceedings of the 2017 CHI Conference on Human Factors in Computing Systems (CHI '17). ACM, New York, NY, USA, 1339-1351. https://doi.org/10.1145/3025453.3025715

[25] Stefan Manojlovic, Laurens Boer, and Paula Sterkenburg. 2016. Playful Interactive Mirroring to Support Bonding Between Parents and Children with Down Syndrome. In Proceedings of the The 15th International Conference on Interaction Design and Children (IDC '16). ACM, New York, NY, USA, 548-553. https://doi.org/10.1145/2930674.2935987 
[26] Gabriela Marcu, Anind K. Dey, and Sara Kiesler. 2012. Parent-driven Use of Wearable Cameras for Autism Support: A Field Study with Families. In Proceedings of the 2012 ACM Conference on Ubiquitous Computing (UbiComp '12). ACM, New York, NY, USA, 401-410. https://doi.org/10.1145/2370216.2370277

[27] Melissa Mazmanian and Simone Lanette. 2017. Okay, one more episode: An ethnography of parenting in the digital age. In Proceedings of the 2017 ACM Conference on Computer Supported Cooperative Work and Social Computing. ACM, 2273-2286.

[28] Andrew D. Miller, Sonali R. Mishra, Logan Kendall, Shefali Haldar, Ari H. Pollack, and Wanda Pratt. 2016. Partners in Care: Design Considerations for Caregivers and Patients During a Hospital Stay. In Proceedings of the 19th ACM Conference on Computer-Supported Cooperative Work \& Social Computing (CSCW '16). ACM, New York, NY, USA, 756-769. https://doi.org/10.1145/2818048.2819983

[29] Annemarie Mol. 2008. The logic of care: Health and the problem of patient choice. Routledge.

[30] Annemarie Mol, Ingunn Moser, Jeannette Pols, et al. 2010. Care: putting practice into theory. Care in practice: On tinkering in clinics, homes and farms 8 (2010), 7-27.

[31] Wendy Moncur, Judith Masthoff, Ehud Reiter, Yvonne Freer, and Hien Nguyen. 2014. Providing adaptive health updates across the personal social network. Human-Computer Interaction 29, 3 (2014), 256-309.

[32] Francisco Nunes and Geraldine Fitzpatrick. 2018. Understanding the Mundane Nature of Self-care: Ethnographic Accounts of People Living with Parkinson's. In Proceedings of the 2018 CHI Conference on Human Factors in Computing Systems (CHI '18). ACM, New York, NY, USA, Article 402, 15 pages. https://doi.org/10.1145/3173574.3173976

[33] Laura R. Pina, Sang-Wha Sien, Teresa Ward, Jason C. Yip, Sean A. Munson, James Fogarty, and Julie A. Kientz. 2017. From Personal Informatics to Family Informatics: Understanding Family Practices Around Health Monitoring. In Proceedings of the 2017 ACM Conference on Computer Supported Cooperative Work and Social Computing (CSCW'17) ACM, New York, NY, USA, 2300-2315. https://doi.org/10.1145/2998181.2998362

[34] Lyndsey Runaas, David Hanauer, Molly Maher, Evan Bischoff, Alex Fauer, Tiffany Hoang, Anna Munaco, Roshun Sankaran, Rahael Gupta, Sajjad Seyedsalehi, et al. 2017. BMT roadmap: a user-centered design health information technology tool to promote patient-centered Care in Pediatric Hematopoietic Cell Transplantation. Biology of Blood and Marrow Transplantation 23, 5 (2017), 813-819.

[35] Anselm Strauss and Juliet M Corbin. 1997. Grounded theory in practice. Sage.

[36] Anselm L. Strauss, Shizuko Y. Fagerhaugh, Barbara Suczek, and Carolyn L. Wiener. 1997. Social organization of medical work. Transaction Publishers.

[37] Hyewon Suh, John R. Porter, Alexis Hiniker, and Julie A. Kientz. 2014. @BabySteps: Design and Evaluation of a System for Using Twitter for Tracking Children's Developmental Milestones. In Proceedings of the 32Nd Annual ACM Conference on Human Factors in Computing Systems (CHI '14). ACM, New York, NY, USA, 2279-2288. https: //doi.org/10.1145/2556288.2557386

[38] Hyewon Suh, John R Porter, Robert Racadio, Yi-Chen Sung, and Julie A Kientz. 2016. Baby Steps Text: Feasibility Study of an SMS-Based Tool for Tracking Children's Developmental Progress. In AMIA Annual Symposium Proceedings, Vol. 2016. American Medical Informatics Association, 1997.

[39] Matthieu Tixier, Gérald Gaglio, and Myriam Lewkowicz. 2009. Translating Social Support Practices into Online Services for Family Caregivers. In Proceedings of the ACM 2009 International Conference on Supporting Group Work (GROUP '09). ACM, New York, NY, USA, 71-80. https://doi.org/10.1145/1531674.1531685

[40] Tammy Toscos, Kay Connelly, and Yvonne Rogers. 2012. Best intentions: health monitoring technology and children. In Proceedings of the SIGCHI conference on Human Factors in Computing Systems. ACM, 1431-1440.

[41] Naomi Yamashita, Hideaki Kuzuoka, Keiji Hirata, and Takashi Kudo. 2013. Understanding the Conflicting Demands of Family Caregivers Caring for Depressed Family Members. In Proceedings of the SIGCHI Conference on Human Factors in Computing Systems (CHI '13). ACM, New York, NY, USA, 2637-2646. https://doi.org/10.1145/2470654.2481365

[42] Naomi Yamashita, Hideaki Kuzuoka, Keiji Hirata, Takashi Kudo, Eiji Aramaki, and Kazuki Hattori. 2017. Changing Moods: How Manual Tracking by Family Caregivers Improves Caring and Family Communication. In Proceedings of the 2017 CHI Conference on Human Factors in Computing Systems (CHI '17). ACM, New York, NY, USA, 158-169. https://doi.org/10.1145/3025453.3025843

[43] Bridget Young, Mary Dixon-Woods, Michelle Findlay, and David Heney. 2002. Parenting in a crisis: conceptualising mothers of children with cancer. Social Science Medicine 55, 10 (2002), 1835-1847. https://doi.org/10.1016/s0277-9536(01) 00318-5

[44] Bridget Young, Mary Dixon-Woods, and David Heney. 2002. Identity and role in parenting a child with cancer. Pediatric Rehabilitation 5, 4 (2002), 209-214. https://doi.org/10.1080/1363849021000046184

Received April 2019; revised June 2019; accepted August 2019

Proc. ACM Hum.-Comput. Interact., Vol. 3, No. CSCW, Article 153. Publication date: November 2019. 